\title{
Primary immune thrombocytopenia in adults: Belgian recommendations for diagnosis and treatment anno 2021 made by the Belgian Hematology Society.
}

\section{A. Janssens, D. Selleslag, J. Depaus, Y. Beguin \& C. Lambert}

To cite this article: A. Janssens, D. Selleslag, J. Depaus, Y. Beguin \& C. Lambert (2021): Primary immune thrombocytopenia in adults: Belgian recommendations for diagnosis and treatment anno 2021 made by the Belgian Hematology Society., Acta Clinica Belgica, DOI: 10.1080/17843286.2021.1876310

To link to this article: https://doi.org/10.1080/17843286.2021.1876310

曲 Published online: 26 Feb 2021.

Submit your article to this journal $₫$

山 Article views: 122

Q View related articles $\sqsubset$

View Crossmark data \lceil 


\title{
Primary immune thrombocytopenia in adults: Belgian recommendations for diagnosis and treatment anno 2021 made by the Belgian Hematology Society.
}

\author{
A. Janssens ${ }^{a}$, D. Selleslag ${ }^{b}$, J. Depausc, Y. Beguin ${ }^{d}$ and C. Lambert ${ }^{\mathrm{e}}$ \\ ${ }^{a}$ Department of Hematology, Universitaire Ziekenhuizen Leuven, Leuven, Belgium; bepartment of Hematology, AZ Sint-Jan Brugge, \\ Brugge, Belgium; 'Department of Hematology, CHU UCL Namur, Yvoir, Belgium; dDepartment of Hematology, Centre Hospitalier \\ Universitaire Liège, Liège, Belgium; 'Department of Hematology, Cliniques Universitaire St Luc, Bruxelles, Belgium
}

ABSTRACT

The Belgian Hematology Society (BHS) updated the 2013 guidelines for diagnosis and treatment of primary immune thrombocytopenia (ITP) [1]. As knowledge about ITP pathophysiology is increasing, the mode of action of old therapies is better understood and novel drugs are introduced to target more specific pathways.

Corticosteroids with or without intravenous immunoglobulins (IgIV) remain the first-line treatment. According to the updated international guidelines a short course of corticosteroids rather than a prolonged treatment has to be recommended. The same guidelines stress that consequent therapies as thrombopoietic agents (TPO-RAs) and rituximab should be available independent of duration of ITP.

Although the majority of recommendations is based on very low-quality evidence, it is strongly advised to individualize the ITP management taking patient values. and preferences in account. The main treatment goal in all ITP patients must be to maintain a safe platelet count to prevent or stop bleeding with a minimum of toxicity and not to normalize the platelet count.
KEYWORDS

Primary immune thrombocytopenia; diagnosis; treatment; bleeding; pregnancy

\section{Introduction}

By definition immune thrombocytopenia is an acquired autoimmune disease characterized by an isolated low platelet count number $\left(<100 \times 10^{9} / \mathrm{L}\right)$. The disorder is classified as primary immune thrombocytopenia (ITP) in the absence of any obvious initiating and/or underlying cause, and as secondary ITP in association with autoimmune disorders (systemic lupus erythematosus (SLE), the antiphospholipid syndrome (APS), ...), some immunodeficiency syndromes (common variable immunodeficiency syndrome (CVID), adult lymphoproliferative syndrome, ...), lymphoproliferative disorders (chronic lymphocytic leukemia, Hodgkin's disease, large granular T-lymphocyte proliferation, ...), persistent infections (human immunodeficiency virus (HIV), hepatitis $C$ (hep C) virus or Helicobacter $(H)$ pylori) and vaccination $[1,2]$. Several retrospective analyses confirm that approximately $80 \%$ of immune thrombocytopenia's have a primary and $20 \%$ a secondary origin [3,4]. Even today, as no reliable test to diagnose ITP exist, ITP remains a diagnosis of exclusion. In one ITP register, misdiagnosis was seen in 1 out of 7 suspected primary ITP patients [5]. In patients refractory to $\geq 2$ treatments the percentage of primary ITP goes even down to $50 \%$ meaning that diagnosis must be strongly reconsidered in refractory patients. Myelodysplasia, a bone marrow failure syndrome, an inherited thrombocytopenia or a secondary ITP are mostly responsible for the thrombocytopenia in the other $50 \%$ of difficult to treat patients [6]. ITP is further defined as newly diagnosed (duration less than 3 months after diagnosis), persistent (duration 3 to 12 months when spontaneous remission is not reached or complete response (CR) to therapy is not maintained) and chronic ITP (duration more than 12 months) [1].

\section{Incidence}

ITP affects all age groups. Although there are still differences between pediatric ITP (abrupt onset, more bleeding symptoms, higher spontaneous remission rate) and adult ITP (more comorbidities, higher risk of intracranial haemorrhage, likelihood of chronicity), many similarities are found especially between the older children, adolescents and young adults [7]. Data from the pediatric and adult registry on chronic ITP showed that a platelet count of $>100 \times 10^{9} / \mathrm{L}$ was reached in $71 \%$ of children and $49 \%$ of adults 12 months after diagnosis [8]. The incidence of ITP among men and women differs with age, meaning a male predominance below 5 and above 60 years and a female predominance between 6 and 60 years $[9,10]$. Data on epidemiology show an annual incidence of newly diagnosed ITP among adults ranging from 2 to 4 per 100,000 persons per year and

*CONTACT A. Janssens Email: ann.janssens@uzleuven.be Department of Hematology, UZ Leuven, Herestraat 49, 3000 Leuven,Belgium 
a prevalence up to 10 per 100,000 persons per year $[9,11]$. A recent ITP report from US clinical practice showed an even higher annual incidence of 6.1 per 100,000 persons (all ages) [12]. However, ITP is still a rare disease fulfilling the criteria of an orphan disease (prevalence $<5 / 10,000$ ).

Pathogenesis [13-16] (Figure 1)

New insights in the pathophysiology underlying ITP have taught us that not only accelerated peripheral platelet destruction but also suppression of the production of new platelets can be responsible for the thrombocytopenia. Antibody-loaded platelets bind to macrophages and dendritic cells by the Fc gamma receptors ((FcyRs)) and are removed, primarily in the spleen. These antibody-loaded platelets can also be destroyed by direct complement-mediated cell lysis. A recent analysis showed that complement levels are reduced in a one third of patients and are associated with severe disease [17]. Platelets can also be destroyed by CD8 cytotoxic $T$ cells (direct platelet lysis, platelet apoptosis due to granzyme $B$, platelet desialyation with hepatic removal). The macrophages after ingesting the antibody-loaded platelets act as antigen-presenting cells and expose platelet epitopes which stimulate CD4 helper T cells with a Th1 phenotype which activate $B$ cells to produce platelet specific autoantibodies. This process is predominantly taking place in the spleen. During active disease, it has been shown that the CD4 cells with a Th1 phenotype are increased, regulatory $T$ cells responsible for self-tolerance decreased and regulatory $\mathrm{B}$ cells regulating antibody production also reduced. During ITP remission (spontaneous or treatment free-remission (TFR)) CD4 T cells show a Th2/3 phenotype and regulatory $\mathrm{T}$ and $\mathrm{B}$ cells are restored. Platelet production is frequently reduced because autoantibodies against platelet glycoproteins also bind to megakaryocytes, interfere with their maturation and lead to apoptosis with reduced platelet shedding. More recently, it has been shown that megakaryocytes can be destroyed also by CD8 cytotoxic T cells. Besides, the level of thrombopoietin (TPO), the primary growth factor for the regulation of platelet production, in ITP is relatively low compared to patients with thrombocytopenia's resulting from megakaryocytic hypoplasia. This can be explained first by the fact that TPO production is suboptimal as the Ashwell-Morell receptors in hepatocytes are not triggered enough by the absence of old, desialyated platelets and second by the exaggerated removal of TPO bound to platelets. This means that the primary underlying mechanism may vary from patient to patient explaining why the response to treatment also differs between patients.

\section{Clinical presentation [18]}

Many patients remain asymptomatic with the diagnosis of ITP made only after a routine blood test. Others may have bleeding symptoms ranging from skin bleeding (petechiae, purpura, bruises) (dry purpura) to mucosal bleeding (gingival bleeding, mouth blisters, epistaxis, blood in stool or urine, heavy menstrual bleeding) or intracranial bleeding (wet purpura) appearing spontaneously or after trauma. Bleeding symptoms have mostly been reported in patients with a platelet count of less than $30 \times 10^{9} / \mathrm{L}$ while life-threatening bleedings are rare in patients with platelets higher than $10 \times 10^{9}$ / L. Other factors influencing the bleeding risk are age, comorbidities, lifestyle, need for invasive

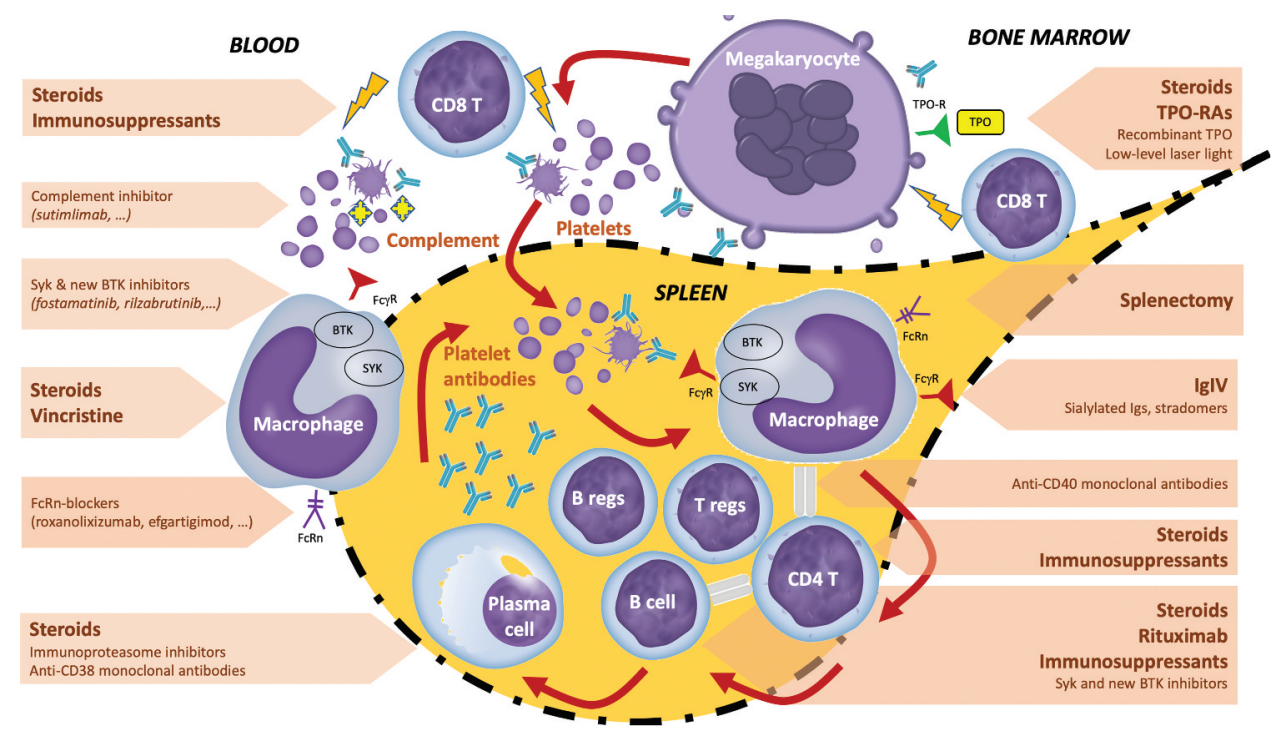

Figure 1. Pathophysiology of ITP and mode of action of current and future treatments. Legend: B regs: regulatory B cells, BTK: Bruton tyrosine kinase,CD4 T: CD 4 T cell, CD 8 T: CD8 T cell, FcR: Fc receptor, FcRn: neonatal Fc receptor, lgs: immunoglobulins, IgIV: intravenous immunoglobulins, Syk: spleen tyrosine kinase,TPO: thrombopoitin, TPO-R: thrombopoitin receptor, TPO-RAs: thrombopoitin receptor agonists, $T$ regs: regulatory $T$ cells 
procedures, need of treatment with anticoagulant or antiplatelet agents, ...

\section{Consequences of ITP diagnosis}

The diagnosis and treatment of ITP have a major impact on the daily activities and a major negative impact on quality of life. Questionnaires have shown that the quality of life was at least as worse as patients with diabetes [19]. The more recent ITP world impact survey showed that symptoms as fatigue and anxiety were reported with a high severity in a high number of patients not only at diagnosis but also at follow-up [20]. The disease has also a major impact on the healthcare (treatment costs) and socio-economic (family, employment) system [21].

\section{- Diagnostic work up [2,22-24]}

\section{Basic evaluation}

- Personal history (prescription and nonprescription drugs, consumption of beverages (tonic water, bitter lemon), food (tahini \& sesam seeds, lupus beans), Jui herbal (Chinese) tea, herbal products, ... (http://www.ouhsc.edu/platelets/ ditp.html); alcohol abuse, recent transfusions, ...)

- Familial history (to exclude inherited thrombocytopenias as Von Willebrand disease type 2B (VWD type 2 B), MYH9 associated syndromes, ...)

- Physical examination (with attention to nodebearing areas, size of liver and spleen)

A complete blood count with peripheral blood smear examination (platelet count measured not only on EDTA but also heparin and citrate anticoagulated blood) (to exclude platelet aggregates (pseudo-thrombocytopenia), platelet aberrations (giant platelets, ...), red cell abnormalities (fragmentocytes, agglutination, microspherocytes, nucleated progenitors, ...) and white cell abnormalities (neutrophilia, inclusions, progenitors, dysplastic changes, lymphocytosis, atypical lymphocytes, ...)

- Immunoglobulin levels (to exclude CVID)

- Direct antiglobulin test (positive in 20\% of ITP) and reticulocytes (exclude Evan's syndrome)

Virology: HIV, hep C, hep B

- H. pylori (antigen in stool or urea breath test) (in patients with digestive symptoms and/or living in geographical areas with high prevalence)

- Blood group (for transfusion policy)

- Bone marrow aspirate and biopsy (with immunophenotyping, karyotyping, NGS panels for myelodysplasia, inherited thrombocytopenia and bone marrow failure syndrome) (only in selected patients with aberrant physical examination or blood smear abnormalities or refractory to treatment)

\section{Tests of potential utility}

Coagulation tests (basic \pm additional tests) (to exclude DIC, VWD type 2B, thrombotic microangiopathy if suspected signs or symptoms)

Antinuclear antibodies (positive in $\pm 33 \%$ of ITP)

Antiphospholipid antibodies (anti-cardiolipin, antibeta 2 glycoprotein I, lupus anticoagulant) (positive in 20 to $40 \%$ of ITP) (to exclude APS if history of arterial/ venous thrombosis or fetal loss))

Thyroid function with antithyroid antibodies (to exclude thyroid dysfunction)

Pregnancy test in women of childbearing potential (to exclude gestational thrombocytopenia)

Anti-platelet antibodies (high degree of variability in the diagnostic test characteristics (sensitivity and specificity) of the available assays)

Virology: PCR for Cytomegalovirus, Epstein-Barr virus, parvovirus B19

Imaging: chest radiograph, abdominal ultrasound, CT-scans or PET-scan (to exclude chest, abdominal organ pathology, such as portal hypertension and splenomegaly ...)

\section{Tests of unproven utility}

Serum complement (optimal assay(s) to be confirmed), serum TPO, immature platelet fraction

Indications for initiation of treatment [22,25]

Treatment is mandatory in patients with 'active bleeding' OR 'platelets $<10 \times 10^{9} / \mathrm{L}^{\prime}$.

Treatment is generally recommended in patients with 'No or mild bleeding' AND 'platelets 10-30 $x 10^{9} / L^{\prime}$. Observation is a potential option in selected patients after evaluation of patient characteristics (age, comorbidities, previous bleeding history, preference, lifestyle, work, pregnancy, ...)

No need for treatment unless special circumstances (previous bleeding history, lifestyle, work, invasive procedures, concomitant anticoagulant or antiplatelet agents, ...) in patients with 'No bleeding' AND 'platelets $>30 \times 10^{9} / L^{\prime}$.

\section{Treatment}

The main treatment goal in all ITP patients must be to maintain a safe platelet count to prevent or stop bleeding and not to normalize the platelet count, to decrease activity restrictions and improve quality of life with a minimum of exposure to potentially toxic therapy [25]. In the past we have seen that morbidity 
Table 1. Safe platelet count for medical interventions in patients with platelet production problems $[23,24]^{*}$

\begin{tabular}{|c|c|c|}
\hline Dental care: & $\geq 10-20 \times 10^{9} / L$ & \multirow{19}{*}{$\geq 40-50 \times 10^{9} / \mathrm{L}$ move to other colum } \\
\hline Extractions (simple): & $\geq 30 \times 10^{9} / \mathrm{L}$ & \\
\hline Extractions (complex, molar): & $\geq 50 \times 10^{9} / \mathrm{L}$ & \\
\hline Lumbar puncture: add elective here & elective delete & \\
\hline vital indication add lumbarpuncture: vital indication & $\geq 20 \times 10^{9} / \mathrm{L}$ & \\
\hline Central venous catheter insertion & $\geq 20 \times 10^{9} / \mathrm{L}$ & \\
\hline GI endoscopy with biopsy: & $\geq 20 \times 10^{9} / \mathrm{L}$ & \\
\hline Bronchoscopy: & $\geq 20 \times 10^{9} / \mathrm{L}$ & \\
\hline Bronchoscopy with biopsy & $\geq 50 \times 10^{9} / \mathrm{L}$ & \\
\hline Joint puncture: & $\geq 20 \times 10^{9} / \mathrm{L}$ & \\
\hline Organ biopsy: & $\geq 50 \times 10^{9} / \mathrm{L}$ & \\
\hline \multicolumn{2}{|l|}{ (<for bone marrow biopsy) place this after organ biopsy (previous row) } & \\
\hline Minor surgery: & $\geq 50 \times 10^{9} / \mathrm{L}$ & \\
\hline Delivery & $\geq 50 \times 10^{9} / \mathrm{L}$ & \\
\hline Major surgery (including neurosurgery): & $\geq 80 \times 10^{9} / \mathrm{L}$ & \\
\hline Epidural anesthesia: & $\geq 70 \times 10^{9} / \mathrm{L}$ & \\
\hline Major neurosurgery & $\geq 100 \times 10^{9} / \mathrm{L}$ & \\
\hline Single antiplatelet or anticoagulant & $\geq 30-50 \times 10^{9} / \mathrm{L}$ & \\
\hline Dual antiplatelet and anticoagulant & $\geq 50-70 \times 10^{9} / \mathrm{L}$ & \\
\hline
\end{tabular}
evidence-based data » and must be individualized to the patient bleeding history

and mortality in ITP was related as much to infection as to bleeding [26].

Before initiating treatment, consideration must be given to:

patient-related factors such as age, performance status, comorbidities, lifestyle (sedentary versus active)

disease-related factors such as platelet count and previous major bleeding

additional risk factors for bleeding such as the use of antiplatelet agents and anticoagulation, uremia, poorly controlled hypertension, aneurysm, fever, chronic liver disease, history of peptic ulcer,

...

treatment-related factors such as expected response, contraindications to and expected sideeffects

the need of invasive procedures (Table 1)

easy access to care facilities

and not at least patient preferences or wishes

\section{When is hospitalization of ITP justified?}

The 2019 updated International Consensus report advise to admit patients with active bleeding or medical conditions which predict a higher bleeding risk or if platelets are $\leq 20 \times 10^{9} / \mathrm{L}$ [23]. The recent ASH guidelines give only a conditional recommendation for admission to the hospital rather than treatment as an outpatient in newly diagnosed patients with platelets $\leq 20 \times 10^{9} / \mathrm{L}$ and no or mild mucocutaneous bleeding [27]. In daily clinical practice, the decision to hospitalize a patient with ITP is based not only on platelet count but on the presence of bleeding symptoms and on factors influencing the bleeding risk as age, comorbidities, need of treatment with anticoagulant or antiplatelet agents, uncertainty of diagnosis, newly diagnosed vs. chronic disease, refractoriness of the disease, easy access to care facilities, social concerns, ...
Treatment of newly diagnosed ITP [23,24,27] (Figure 1-2)

Newly diagnosed ITP patients are managed with corticosteroids with or without intravenous immunoglobulins (IgIV) depending on the severity of thrombocytopenia and/or of the bleeding signs and symptoms. Although impressive responses are seen using these agents, the responses are usually shortlived. Thus, in adults, ITP is very frequently characterized by relapse upon tapering or discontinuation of treatment (only 30 to $50 \%$ of sustained response after discontinuation of corticosteroids) [8,28-30]. Today, prolonged exposure to corticosteroids is no longer recommended because of long-term adverse events. The recent 2019 updated International Consensus report and 2019 ASH ITP guidelines strongly recommend a short course of prednisone (or equivalent) (6 to 8 weeks treatment, tapering included) rather than prolonged treatment. The aim is to stop corticosteroids in a short period of time, not to increase the dose when platelets are dropping again and also to avoid a low dose chronically. In a meta-analysis of randomizedcontrolled trials (RCTs) comparing pulses of high-dose dexamethasone versus (vs.) prolonged use of prednisone or equivalents, faster platelet responses (day 14) and higher response rates were seen for dexamethasone but sustained response ( 6 months) was not different. Changes in facial and body features were more important with prednisone or equivalents as neuropsychiatric side effects were more frequent with dexamethasone [31]. Therefore, both updated ITP guidelines suggest either dexamethasone or prednisone (or equivalent) as the type of corticosteroid for initial treatment. In Belgium prednisone is not available, prednisolone and methylprednisolone have to be used. Characteristics of treatment with corticosteroids and IgIV are shown in Tables 2 and 3.

Platelet transfusions are 'only' exceptionally indicated in case of life-threatening bleeding 


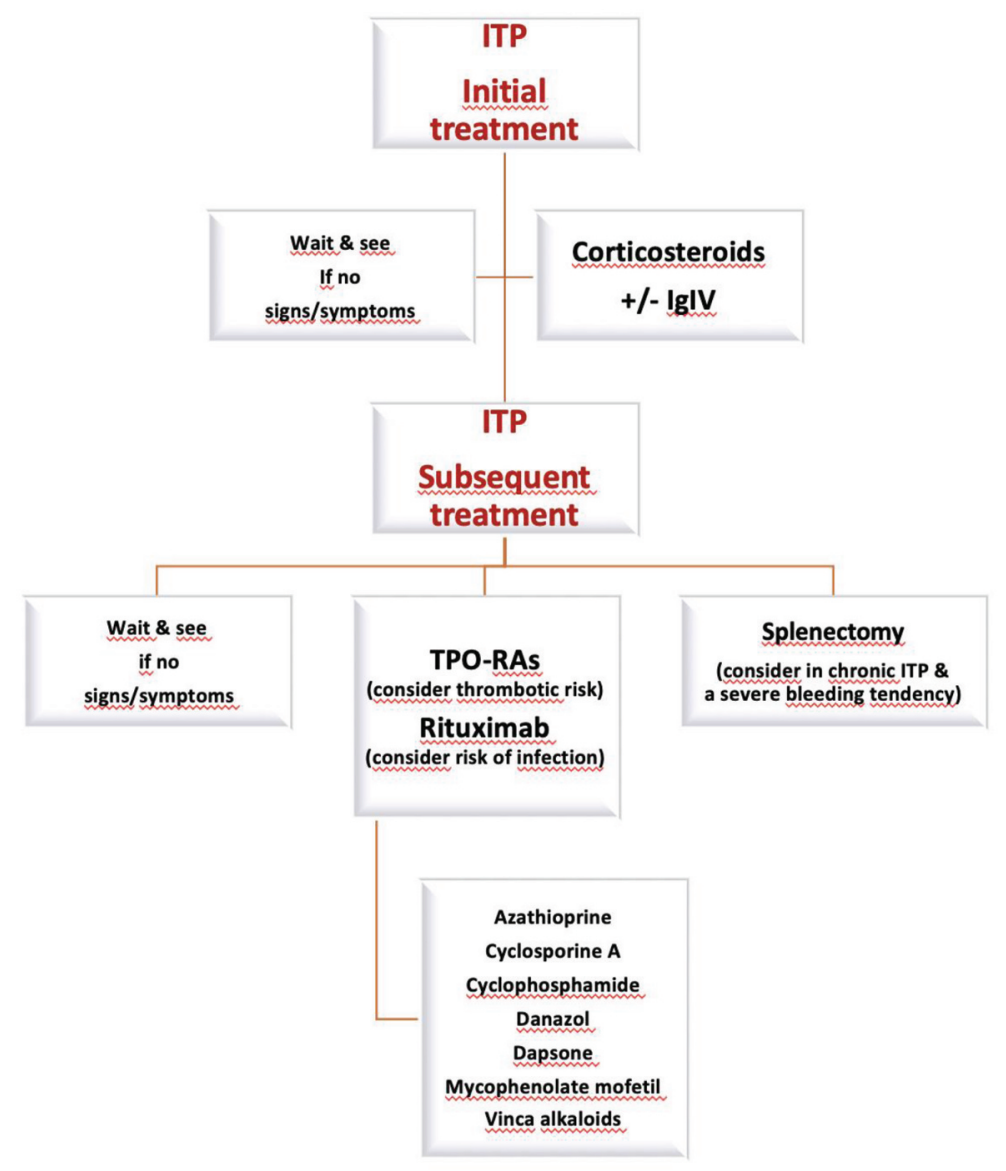

Figure 2. Treatment algorithm for adult ITP.

Table 2. Characteristics of treatment with corticosteroids

No preference to choose (methyl)prednisolone or dexamethasone (dexamethasone has a longer half-life, no mineralocorticosteroid effect, a potent anti-plasma cell effect and provides a faster platelet response)

(Methyl)prednisolone or equivalent: continuous treatment for 6-8 weeks

Start dose: $1 \mathrm{mg} / \mathrm{kg}$ (maximum of $80 \mathrm{mg} / \mathrm{d}$ ) prednisolone) for 7-14 days

Tapering: slow tapering (5-7 weeks) during the following weeks if responsive, rapid tapering (in 1 week) if not responsive Dexamethasone: $40 \mathrm{mg} / \mathrm{d}$ for 4 days, to be repeated after 15 or 28 days for 1 to 3 cycles, depending on the response

Short term response of corticosteroids: $\pm 80 \%$

Durable response of corticosteroids: $30-50 \%$

Mode of action: modulates T cells (restoring Th1/Th2 ratio, T regs) modulates $B$ cell activation (B cells, $B$ regs, plasma cells) and modulate DCs which lead to impairment of antibody production, clearance of antibody loaded platelets by phagocytes, increase of thrombopoiesis, improvement vascular integrity, ...

Side effects: changing facial and body features, arterial hypertension, myopathy, diabetes mellitus, psychological problems, osteoporosis, osteonecrosis, cataract, glaucoma, ...

More side effects compared with other therapies leading to dose reduction and treatment discontinuation

Absolute contraindication for steroids: acute viral infection (Herpes simplex or Herpes zoster, ...), hep B s Ag positive, chronic active hepatitis

Relative contraindication for steroids: peptic ulcers, bacterial

infections, history of tuberculosis, mycosis, parasitosis, uncontrolled hypertension, diabetes mellitus, osteoporosis, psychosis, glaucoma, and recent entero-anastomoses

Cheap

together with the administration of corticosteroids and IgIV. Platelet transfusions are frequently needed
Table 3. Characteristics of treatment with intravenous immunoglobulins (IgIV)

Recommended dose: $400 \mathrm{mg} / \mathrm{kg} / \mathrm{d}$ for 5 days or $1 \mathrm{~g} / \mathrm{kg} / \mathrm{d}$ for 1 or 2 days
Short term response: $\pm 80 \%$, rapid response
Durable response: exceptional, relapse between $14-28 \mathrm{~d}$
Repeated infusions possible
Mode of action: impairs clearance of antibody loaded platelets by
macrophages by saturating the (FcyRs) on macrophages, promoting
the expression of inhibitory FcyRllb, saturation of the FcRn which
increases the clearance of autoreactive antibody, modulation of
T and B cells (impairment of B cell function, increase of T suppressors)
Side effects: infusion related effects (can be managed with
concomitant steroid premedication), headache, aseptic meningitis,
renal impairment (only for preparations containing sucrose),
thromboembolic events
Subcutaneous (sc) Ig does not appear to be an alternative to IgIV
Expensive

in this situation. Only in patients with lifethreatening bleeding not responding to the previous mentioned measures to achieve hemostasis, rituximab and/or TPO-receptor agonists (TPO-RAs) can be added.

Treatment of persistent ITP (lasting 3 to 12 months after diagnosis) and chronic ITP (lasting >12 months after diagnosis) [23,24,27] (Figure 1-2)

Indications for starting treatment in persistent and chronic ITP are identical to those of newly diagnosed ITP. Relapse after a long treatment-free interval can be managed by restarting first-line treatment being steroids and/or IgIV if the patient was responsive before. 
Table 4. Treatment options for relapsing and refractory adult ITP.

\begin{tabular}{lll}
\hline \multicolumn{1}{c}{ dose } & \\
\hline Azathioprine & $1-2 \mathrm{mg} / \mathrm{kg} / \mathrm{d}$ orally & Neutropenia, transaminase elevation, pancreatitis, ... \\
Cyclophosphamide & $1-2 \mathrm{mg} / \mathrm{kg} / \mathrm{d}$ orally & Nausea, vomiting, sterility, secondary AML, ... \\
Cyclosporine & $4-5 \mathrm{mg} / \mathrm{kg} / \mathrm{d}$ orally & Renal insufficiency, hypertension, neuropathy, hypertrichosis, tremor, gingival hyperplasia, ... \\
$\begin{array}{l}\text { Danazol } \\
\text { Dapsone }\end{array}$ & $400-800 \mathrm{mg} / \mathrm{d}$ orally & Weight gain, hair loss, liver dysfunction, myalgia, amenorrhea, ... \\
& $75-100 \mathrm{mg} / \mathrm{d}$ orally & Abdominal distension, anorexia, nausea, rash, hemolytic anemia if G6PD deficiency, ... \\
Mycophenolate mofetil & (not after splenectomy) & \\
$\begin{array}{l}\text { Vincristine } \\
\quad \text { Vinblastine }\end{array}$ & $1000 \mathrm{mg} 2 \mathrm{x} / \mathrm{d}$ orally & Headache, anorexia, nausea, abdominal distention, .... \\
\hline
\end{tabular}

As for newly diagnosed patients, again, a long-term steroid treatment or a low dose of steroids chronically must be avoided.

A subsequent therapy is indicated for those patients unresponsive to, relapsing during or shortly after tapering of steroids. The most robust data are available for splenectomy as surgical therapy and the TPO-RAs and rituximab as medical therapies (Table 4).

\section{Splenectomy}

For decades splenectomy has been the standard management for ITP patients with persistent low platelets and a high bleeding risk, unresponsive or intolerant to corticosteroids. Historical data has shown that twothirds of patients could, attain a durable remission. Morbidity and mortality of laparoscopic splenectomy are low in the hands of experienced surgeons. The incidence of overwhelming sepsis is reduced with recommended vaccination protocols and antibiotics initiated at first sign of a systemic febrile illness. However, the last decades, physicians and patients frequently opt to postpone the removal of this healthy organ. As spontaneous remission of ITP up to 12 months after diagnosis can occur in \pm 30 à $50 \%$ adults, an attempt should be made to delay splenectomy until after this point. Ideally, when possible, platelet count should be above $50 \times 10^{9} / \mathrm{L}$ before surgery to reduce complications as mentioned by the 2019 updated International consensus report. Above the age of 60 , higher rates of relapses and more postoperative complications are seen with splenectomy compared to ITP patients under the age of 60 [32]. Characteristics of splenectomy with relative contraindications are shown in Table 5 and 6.

\section{Rituximab}

Rituximab has been used for treating relapsing and refractory ITP patients for almost 20 years. As RCTs are not available to establish optimal dose, schedule and timing of rituximab administration and to confirm efficacy and long-term safety, rituximab has still no license for treating ITP [33]. The recently published
French prospective ITP-ritux registry ( $n=248$, persistent and chronic ITP) confirmed data from previously published cohorts and systemic reviews [34,35]. An overall (ORR) and complete response rate (CR) of approximately $61 \%$ and $32 \%$ has been shown. At 60 months of follow-up, $29.4 \%$ had a sustained response defined as a platelet count of $>30 \times 10^{9} / \mathrm{L}$ without any additional treatment and without any bleeding. The only predictor of sustained response seemed to be previous response to steroids. This registry focused on long-term safety. The incidence of severe infections was less than $10 \%$. Predominantly older patients with contributing factors were affected and $1 / 3$ of the infections occurred in the first year after treatment. Opportunistic infections appeared exceptional with no case of progressive multifocal leukoencephalopathy. Profound late-onset neutropenia was observed in $<1 \%$ of patients. The registry showed that hypogammaglobulinemia could occur even several years after rituximab and need to be monitored. No excess of secondary malignancies, venous thrombosis or cardiovascular events was noticed. Only $5 \%$ of patients developed another autoimmune disease. No difference in short- and long-term responses between the lymphoma rituximab regimen (4 intravenous infusions at $375 \mathrm{mg} / \mathrm{m}^{2}$ weekly) or the rheumatoid arthritis regimen (two fixed $1 \mathrm{~g}$ rituximab infusions 2 weeks apart) was observed. Retreatment with rituximab in responding patients was feasible with $92 \%$ of patients responding again with at least the same response (CR or partial response (PR)). Responses seemed comparable for splenectomized and non-splenectomized patients [35].

\section{Thrombopoietic agents}

The efficacy data of second-generation thrombopoietic agents (TPO-RAs as romiplostim, eltrombopag and avatrombopag) have confirmed the hypothesis that stimulating platelet production is a valid treatment approach in ITP [36-39]. TPO-RAs have been associated with more durable platelet responses and less treatment failure compared to placebo or standard of care in adult chronic ITP patients (defined in the pivotal 
trials as ITP lasting $>6$ months) regardless of splenectomy status. Efficacy data are also identical for patients treated with the TPO-RAs in the acute or persistent ITP phase [40-42]. The response rates obtained in the pivotal studies were reproduced in open-label studies and real-life enrolling patients with more severe comorbidities and older age. Patients relapsing after or refractory to rituximab seem to be as responsive as cohorts were previous exposure to rituximab was less. Treatment with TPO-RAs result in a reduction of bleeding events, in less use of rescue medication or splenectomy and permit the majority of patients receiving concurrent ITP drugs to reduce or discontinue these therapies and avoid further immunosuppression. An additional role of the TPO-RAs may be to use the drug intermittently around the time of an anticipated bleeding risk. TPO-RAs are very well tolerated. As the experience with TPO-RAs in the clinic is exceeding 12 years, we suppose that possible risks of long-term use of TPO-RAs are almost known [37,38]. Characteristics of treatment with TPO-RAs and risks of long-term treatment are shown in Table 7 and Table 8. International guidelines, as well as the regulatory authorities worldwide recommended, suggested and reimbursed romiplostim and eltrombopag as a second or third-line treatment option since 2009 [2,43]. Today, in Belgium, the TPO-RAs, romiplostim and eltrombopag, are reimbursed for the treatment of chronic ITP (ITP lasting >12 months) refractory or intolerant to corticosteroids after splenectomy or when surgery is contraindicated. We are waiting for the extended reimbursements of romiplostim and eltrombopag for persistent ITP once European Medicines Agency labels will have been adjusted. Despite the initial assumption that TPO-RAs would be a life-long therapy, practical experience has shown that a significant number of patients do stop treatment (no efficacy, adverse events, ITP or non-ITP related death or spontaneous remission). Spontaneous remission or TFR is defined as a treatment-free period of $\geq 6$ months with platelets $>50 \times 10^{9} / \mathrm{L}$ and no need of ITP rescue medication. The Belgian Hematology Society (BHS) survey from 2018 showed that TFR was seen in about $22 \%$ of ITP patients treated with TPO-RAs in daily clinical practice in Belgium. TFR was seen not only in acute or persistent but also in chronic ITP and seemed independent of splenectomy and number of treatments given before [44]. In the future, the incidence of TFR is thought to be even higher when the use of a forced tapering schedule will become common practice.

\section{Other medical therapies}

Medical therapies with less robust data for subsequent treatment are azathioprine, cyclophosphamide, cyclosporine A, danazol, dapsone, mycophenolate mofetil and vincristine/vinblastine. These agents have been used after treatment failure in both splenectomized and non-splenectomized patients for decades. These treatment options are characterized by variable individual responses after days, weeks to months and significant long-term side effects such as immune suppression and many others, which must be considered by patient and physician. Most of the retrospective data according these agents show only a response rate of 20 to $50 \%$ with lower response rates in more heavily treated patients and with longer disease duration $[22,45]$. Some characteristics of these agents are shown in Table 4. We presume that most of these agents work by immunosuppression or immunomodulation. Vincristine/vinblastine seem to inhibit the phagocytosis of antibody-loaded platelets by macrophages. For dapsone, it has been postulated that the splenic macrophages are redirected to phagocytosis of the hemolytic-red blood cells with reduction of the platelet phagocytosis.

\section{Novel agents}

Due to better understanding of the pathophysiology novel therapies targeting other pathways are explored. Fostamatinib, the first spleen tyrosine kinase (Syk) inhibitor obtained already Food and Drug Administration approval for ITP patients with an insufficient response to previous treatment. Syk inhibition will reduce antibody-mediated platelet destruction and regulates autoantibody production [46]. The same mode of action is expected for new Bruton tyrosine kinase inhibitors as rilzabrutinib [47]. Phase 2 trial data with roxanolixizumab, a humanized anti-FcRn monoclonal antibody and efgartigimod, a human IgG Fc-fragment blocking the binding of IgG to FcRn confirmed that blocking FcRn is a novel therapeutic approach in ITP by increasing the clearance of antiplatelet antibodies $[48,49]$. Reduction of antibody formation could also be the goal of treatment with anti-CD38, anti-CD40 ligand antibodies and immunoproteasome inhibition. Sialylated IgG and stradomers are tested to reduce platelet phagocytosis. Complement inhibitors could reduce complement-mediated platelet lysis $[6,14,45,50]$.

The BHS recommends the following subsequent treatment options for ITP patients who are intolerant or unresponsive to or relapse after initial corticosteroid treatment and have a risk of bleeding (platelets <30 $\times 10^{9} / \mathrm{L}$ OR bleeding symptoms):

TPO-RAs must be available irrespective of the duration of ITP and the splenectomy status. TPORAs are the best treatment option for patients who prefer a durable platelet response while avoiding surgery and who have an acceptable thrombotic risk.

Rituximab can be used irrespective of the duration of ITP and the splenectomy status. Rituximab is a good 


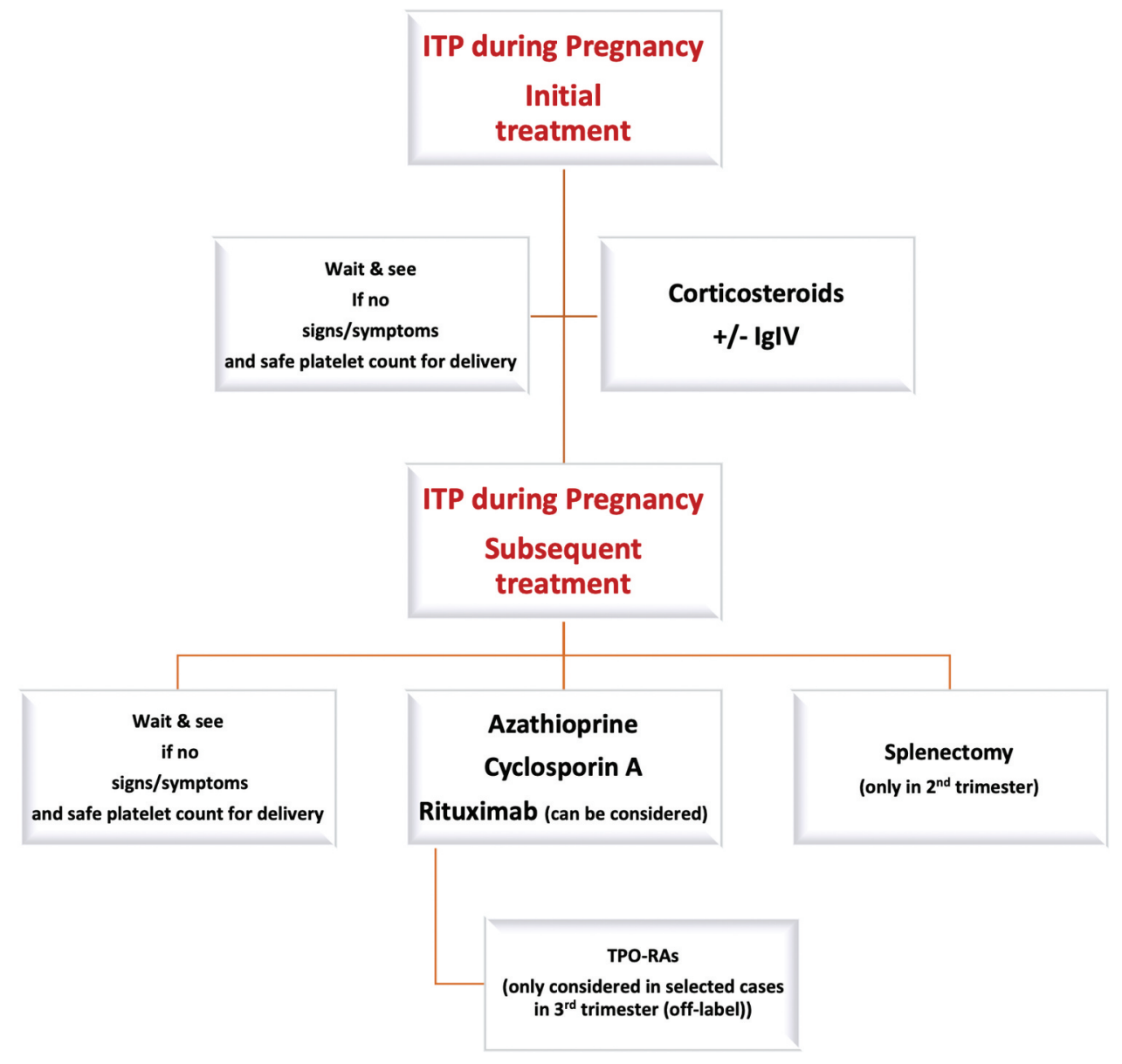

Figure 3. Treatment algorithm for pregnant ITP.

treatment option in patients who want to avoid longterm treatment, surgery and who have an acceptable infectious risk.

Splenectomy is only indicated in patients with a severe bleeding tendency but should be delayed (if possible) to at least 12 months after diagnosis as spontaneous remission can occur in this time period. The best responses and the lowest complication rates are seen in patients $<60$ years. Splenectomy is the best treatment option for patients who prefer a durable platelet response while avoiding long-term medications.

Other medical therapies as azathioprine, cyclophosphamide, cyclosporine A, danazol, dapsone, mycophenolate mofetil and vincristine/vinblastine have to be considered for patients refractory to TPO-RAs, rituximab and/or splenectomy.

ITP in pregnancy [51-54] (Figure 3)

\section{Incidence}

Thrombocytopenia develops in 5 to $10 \%$ of women during pregnancy or in the immediate postpartum period. ITP is an uncommon but important cause of thrombocytopenia in pregnancy ( $3 \%$ of all cases of the thrombocytopenia). The incidence of ITP ranges from 1 to 2 per 1000 pregnancies to 1 per 10000 for severe cases.

\section{Diagnostic work-up}

Diagnosis and work-up are similar to non-pregnant women but differential diagnosis includes pregnancy-specific conditions such as gestational thrombocytopenia, coagulopathy and hypertensive, microangiopathic or hepatic disorders. Those are detailed in Table 9 and must be separated from ITP because their successful management differs completely from that of ITP.

\section{Follow-up and treatment}

Pregnant women with ITP require careful monitoring (monthly till week 28, every 2 weeks till week 36 , weekly till delivery). The indications for treatment of the pregnant woman do not differ from those of a nonpregnant person. However, a platelet count of 50 $\times 10^{9} / \mathrm{L}$ is preferred for vaginal delivery and caesarean section and a platelet count of $\geq 70 \times 10^{9} / \mathrm{L}$ is recommended for epidural anaesthesia. If treatment is necessary, prednisone or equivalent (preferred to pulse dexamethasone which also crosses the placenta) $1 \mathrm{mg} / \mathrm{kg} /$ day (pre-pregnancy weight) should be considered as first-line and gradually titrated to the lowest effective dose. High doses steroids should be avoided in first trimester to prevent congenital anomalies such 
as cleft palate and prolonged high doses because of exaggerated toxicity during pregnancy (gestational diabetes, hypertension, osteoporosis, weight gain and psychosis). IgIV (1 g/kg 1-2 days) are also considered as an appropriate first-line treatment for ITP during pregnancy as they may increase the platelet count faster than steroids. Patients who fail to respond to corticosteroids or IgIV alone may respond to the combination of these agents. Laparoscopic splenectomy can be safely performed in the second trimester. Splenectomy is not recommended in the first trimester due to the risk of induction of premature labour or in the third trimester due to bad visualization of the surgical field. Azathioprine, cyclosporin A and rituximab are not known to be teratogenic. Rituximab has been used in pregnant women but can be associated with prolonged $B$ cell depletion in neonates exposed in utero resulting in delaying the start of vaccination. The effects on pregnancy outcomes and on the fetus with TPO-RAs are unknown and their experience during pregnancy is anecdotal. This treatment should only be considered in late pregnancy if the potential benefit to the mother justifies the potential risk to the fetus.

Care should be coordinated by experienced colleagues from haematology, obstetrics and neonatology. Maternal and neonatal outcomes are generally favourable but severe ITP carries a higher risk of maternal post-partum haemorrhage. Invasive deliveries (forceps or vacuum-assisted) should be avoided. Thromboprophylaxis and anticoagulation should be managed according to standard protocols as ITP does not prevent thrombosis. Although, the minimal platelet count required in this setting still needs to be validated by evidence-based guidelines. Tranexamic acid is a safe and effective adjunct in the peri-partum period. Nonsteroidal anti-inflammatory drugs should be avoided in the post-partum period if the platelet count is $<70 \times 10^{9} / \mathrm{L}$.

\section{Follow-up of the neonate}

With respect to the neonates, platelet counts $<20 \times 10^{9}$ / $\mathrm{L}$ and $<50 \times 10^{9} / \mathrm{L}$ are seen in, respectively, $1-5 \%$ and $10 \%$ and $5-15 \%$ will require therapy. Thrombocytopenia in neonates is due to transplacental passage of antiplatelet antibodies present in mothers with ITP. This can also occur with maternal ITP in remission with or without splenectomy. Neonatal intracranial hemorrhage is seen in $<1 \%$ and is not associated with the mode of delivery. Therefore, the mode of delivery in pregnant ITP patients should be solely based on obstetrical considerations. However, invasive procedures during labor (scalp electrodes, vacuum extractor, forceps) should be avoided as they are associated with an increased hemorrhagic risk to the fetus. Cordocentesis and fetal scalp or percutaneous umbilical blood sampling should also be avoided. A cord platelet count is indicated after delivery and serial platelet counts should be obtained in the newborns at birth and in the first week postpartum as the onset of thrombocytopenia can be delayed. IgIV and platelet transfusions are indicated to manage bleeding or if platelet count $<30 \times 10^{9} / \mathrm{L}$ in the neonates. Cerebral imaging is recommended in newborns with platelets $<50 \times 10^{9} / L$ to exclude an intracranial hemorrhage.

\section{Counselling of women with ITP}

ITP is usually not a contra-indication for pregnancy risk of fetal thrombocytopenia exist even in splenectomised mothers

no correlation between the platelet count of the mother and the newborn.

the most reliable predictor of neonatal thrombocytopenia is a history of low platelet count at delivery in a prior sibling

breastfeeding should not be discouraged.

\section{Supportive treatment for ITP patients}

Antifibrinolytic agents

Oral iron supplements if required

Local application of adrenalin-soaked nose pads

Nasal cauterisation

Hormonal therapy to prevent menorrhagia

Control of blood pressure

Stop or avoid acetylsalicylacid (ASA), nonsteroidal anti-inflammatory drugs, antiplatelet agents, anticoagulation if appropriate

\section{Management of ITP during the COVID-19 pan- demic [55]}

In newly diagnosed patients it seems advisable to avoid corticosteroids or to use the lowest effective dose as possible for the shortest time to not increase the susceptibility to the SARS-CoV-2. IgIV can be used at diagnosis or at relapse. The UK ITP experts even recommend TPO-RAs as first line treatment. Chronic ITP patients should continue their chronic treatment. As ITP, TPO-RAs and COVID-19 infection have all an increased thrombotic risk, ITP patients with a severe COVID-19 infection and treated with TPO-RAs, must receive low molecular weight heparin $(\mathrm{LMWH})$ if platelets $>30 \times 10^{9} / \mathrm{L}$ or calf compression therapies if $<30$ x $10^{9} /$ L. Patients on high-dose steroids for weeks, low dose steroids with another immunosuppressant, rituximab during the last 12 months with another immunosuppressant or corticosteroids, splenectomy with an immunosuppressant and/or concomitant comorbidities as age $>70$ years, diabetes mellitus, pre-existing lung, renal or cardiac disease and hypertension should strongly be advised to minimize the risk of SARS-CoV2 infection by social distancing, wearing protective masks and hygienic measures. Subsequent therapies as rituximab and splenectomy should be postponed, if possible, due to the increased infection risk. 


\section{Conclusion}

Primary ITP is characterized by an isolated low platelet count. The clinical picture (mild to severe bleeds) is as heterogeneous as the pathophysiology of the disorder. Today we know that ITP pathogenesis is associated with $B$ and $T$ cell defects which lead to platelet and megakaryocyte damage resulting in platelet destruction and inefficient thrombopoiesis. Although severe bleeds in adults are not much higher than $10 \%$ [56], the disease has a major impact on daily activities and quality of life. ITP patients has also a higher mortality rate than the general population. This is not only due to bleeding but also to infections and cardiovascular problems. Personalized treatment decisions remain a challenge in many cases since the underlying pathogenesis can differ between patients. The BHS recommends TPO-RAs, rituximab and splenectomy as the subsequent treatment options for ITP patients who are intolerant or unresponsive to or relapse after initial corticosteroid treatment and have a risk of bleeding (platelets $<30 \times 10^{9} / \mathrm{L}$ OR bleeding symptoms). TPO-RAs and rituximab should be used irrespective of the duration of ITP, while splenectomy should be deferred for at least 12 months after diagnosis. Currently, no pathophysiological difference is known for acute, persistent and chronic ITP. Today, we are waiting in Belgium for the extension of the reimbursement of TPO-RAs for patients with an ITP duration of less than 12 months.

- Retreatment options

- Corticosteroids $1 \mathrm{mg} / \mathrm{kg} / \mathrm{d}$ orally (prednisone or equivalent), $40 \mathrm{mg} / \mathrm{d} \times 4 / \mathrm{q} 14-28 \mathrm{~d}, \times 1-3$ orally (dexamethasone)

- Immunoglobulins $400 \mathrm{mg} / \mathrm{kg} / \mathrm{d} \times 5 \mathrm{~d}$ or $1 \mathrm{~g} / \mathrm{kg} / \mathrm{d}$ $x$ 1-2d IV

- Second- or further line treatment options

- Splenectomy

- TPO-RAs

○ Romiplostim 1-10 $\mu \mathrm{g} / \mathrm{kg} / \mathrm{w} \mathrm{sc}$

- Eltrombopag25-75 mg/d orally

- Avatrombopag20 to $40 \mathrm{mg} / \mathrm{d}$ orally to

a minimum of $20 \mathrm{mg} /$ week(not available in

Belgium at publication)

- Rituximab375 mg/m2/w for 4 weeks IV or $1000 \mathrm{mg}$ d 1 and d $15 \mathrm{IV}$

Table 5: Characteristics of splenectomy in adult ITP

As at least $30-50 \%$ of patients remit in the first year after diagnosis, it is recommended to postpone splenectomy till the chronic ITP stage

Most effective durable treatment for adults $( \pm 66 \%$ long-term normal platelets, $\pm 14 \%$ no response, $\pm 20 \%$ late failures)

Perioperative complications (comparable to other minor surgical procedures and general anesthesia)

Mortality: $1 \%$ for laparotomy, $0.2 \%$ for laparoscopy
Morbididy: $13 \%$ for laparotomy, 10\% for laparoscopy

Acute venous thromboembolism: low but increased incidence (appropriate thrombosis prophylaxis postoperative especially when risk factors for thrombosis present, ASA if platelets $>500 \times 10^{9} / \mathrm{L}$ )

Acute portal vein thrombosis: can occur in the first 3 months; range from asymptomatic to fatal; higher after laparoscopy; start diagnostic investigations in patients with cramping abdominal pain, depressed bowel sounds and generalized bowel tenderness

Laparoscopic splenectomy: less postoperative pain, less infectious complications, earlier diet tolerance, shorter hospital stay but more haemorrhagic complications

No preoperative characteristic can predict success of splenectomy ( ${ }^{111}$ In platelet survival: if only splenic sequestration, $90 \%$ response; less response if platelets very low, $>3$ previous treatments, elderly)

Postoperative thrombophylaxis should be considered as long as platelets are $>30-50 \times 10^{9} / \mathrm{L}$ [20]

Long-term effects:

- Overwhelming post-splenectomy infection (S. pneumoniae, H. influenzae, N. meningitidis, E. coli, Capnocytophagia canimorsus, group B streptococcus, Ehrlichia and plasmodium species (cavé travellers!))

- Appropriate and timely immunization (2-4 w before or otherwise $2 \mathrm{w}$ after splenectomy) [57]

- Influenza vaccine: lifelong, once yearly

- Pneumococcal vaccine: one dose PCV13 followed, at least 2 months later, by one dose PPSV23, repeat PPSV23 every 5 years.

- Meningococcal vaccine: one dose quadrivalent conjugated meningococcal vaccine $(A, C, W, Y)$ and two doses meningococcal $B$ vaccine, at least one month apart

- $\mathrm{H}$ influenzae vaccine: one dose if not previously vaccinated

Prophylactic antibiotics in adults only when timely immunization not possible

Education of each patient regarding risk of overwhelming infection life long

Prompt treatment of infection life long

Today, with all mentioned precautions, infection risk after 3 months not higher in splenectomy vs. nonsplenectomy patients

Cost: affordable

Table 6. Relative contraindications for splenectomy

Platelet count $<20 \times 10^{9} / \mathrm{L}$ before surgery (platelets $>50 \times 10^{9} / \mathrm{L}$ are ideal to limit the risk of postoperative bleeding, to minimize trauma to upper airway during general anaesthesia)

Older age ( $>60$ years, higher rates of relapse and more postoperative complications) 
Comorbidities increasing perioperative risk

- Diabetes mellitus

- Heart failure, ischemic heart disease,

- Arrythmias, valvular disease

- Uncontrolled arterial hypertension

- Chronic obstructive pulmonary disease, asthma

- Renal insufficiency $(\mathrm{Cr}>2 \mathrm{mg} / \mathrm{dl})$

- History of stroke

- Severe obesity

Low functional capacity

Refusal by the patient

Table 7. Characteristics of treatment with TPOreceptor agonists (RAs)

Dosing of TPO-RAs

- Romiplostim

o start with $1 \mu \mathrm{g} / \mathrm{kg}$ sc

- Increase dose with $1 \mu \mathrm{g} / \mathrm{kg} / \mathrm{w}$ if platelet count $<50 \times 10^{9} / \mathrm{L}$ till a maximum dose of $10 \mu \mathrm{g} / \mathrm{kg}$

- Maintain dose if platelet count between $50-150 \times 10^{9} / \mathrm{L}$

- Reduce dose with $1 \mu \mathrm{g} / \mathrm{kg}$ if platelet counts between $150-250 \times 10^{9} / \mathrm{L}$ for 2 weeks

- Stop dosing and reassess after 1 week if platelet count $>250 \times 10^{9} / \mathrm{L}$ and restart dosing with $-1 \mu \mathrm{g} / \mathrm{kg}$ if platelet count $<150 \times 10^{9} / \mathrm{L}$

o If platelet count $\geq 50 \times 10^{9} / \mathrm{L}$ for at least 4 weeks without dose adjustment, assess platelet count once monthly

- Consider self-administration or home administration by a nurse or caregiver

- Eltrombopag

- Not eat calcium, iron, magnesium, aluminium rich food $4 \mathrm{~h}$ before and $2 \mathrm{~h}$ after ingestion

o Start with $50 \mathrm{mg}$ orally once daily $(25 \mathrm{mg}$ in patients from East Asia and those with a ChildPugh $\geq 5$ )

- If platelet count $<50 \times 10^{9} / \mathrm{L}$ after 2 weeks, increase dose to $75 \mathrm{mg}$ once daily

○ If platelet count between $50-150 \times 10^{9} / \mathrm{L}$, maintain dose

- If platelet count between $150-250 \times 10^{9} / \mathrm{L}$, reduce to $25 \mathrm{mg}$ once daily

○ If platelet count $>250 \times 10^{9} / \mathrm{L}$, stop dosing, monitor platelet count twice weekly and reinitiate at a daily dose reduced by $25 \mathrm{mg}$ if platelet count $<100 \times 10^{9} / 1$

o If platelet count $\geq 50 \times 10^{9} / \mathrm{L}$ for at least 4 weeks without dose adjustment, assess platelet count once monthly

- Avatrombopag (not available in Belgium)

- No dietary limitations

o Start with 20 mg orally once daily with food

- Asses platelet weekly and titrate to a platelet count between $50-200 \times 10^{9} / \mathrm{L}$
- Maximum dose $40 \mathrm{mg}$ once daily orally

- Minimum dose $20 \mathrm{mg}$ once weekly

Romiplostim binds the extracellular TPO-R domain identical to endogenous TPO; eltrombopag and avatrombopag bind the transmembrane TPO-R domain

TPO-RAs induce durable platelet responses $(60 \%$ stable responses, $25 \%$ variable responses, $15 \%$ minor or no response) and less treatment failures

- regardless of splenectomy status

- regardless of response to rituximab

- in chronic (>6 months) ITP but also in persistent and newly diagnosed patients

TPO-RAs reduce the need of rescue medication (IgIV, splenectomy) and permit to reduce or discontinue concurrent ITP drugs (corticosteroids, ...) and avoid further immunosuppression

TPO-RAs reduce bleeding events

TPO-RAs improve health-related quality of life

TPO-RAs are very well tolerated (mild to moderate headache is most common adverse event $),( \pm 10-15 \%$ discontinuation because of intolerance)

Continuous treatment (romiplostim once weekly sc, eltrombobag and avatrombopag once daily orally)

The risk-benefit balance in a patient with a thrombo-embolic risk should be considered before starting TPO-RA (use with caution in patients with APS and previous thrombotic events)

Switching TPO-RAs: can help patients with poor responses, with large fluctuations, side effects and with route of administration problems

Treatment free remission: $10-30 \%$ of patients (defined as $>50 \times 10^{9} / \mathrm{L}$ lasting as least 6 months after discontinuation of the TPO-RAs)

No consensus on tapering

- Consider tapering in those with a complete response on a low dose and stable platelet count for $>6$ months

- An acceptable tapering scheme could be:

- Romiplostim: reduce dose $\mathrm{x}-1 \mu \mathrm{\mu} / \mathrm{kg}$ each two weeks

- Eltrombopag: reduce dose with $25 \mathrm{mg}$ each 2 weeks to $25 \mathrm{mg}$ each 4 days before interrupting

TPO-RAs are not recommended in pregnancy (insufficient safety data available)

Expensive

Table 8. Possible risks of long-term use of TPOreceptor agonists (RAs)

Loss of efficacy with prolonged stimulation of megakaryopoiesis 
Bone marrow fibrosis

- Follow up of blood cell count and smear regularly

- If loss of response, new cytopenia or new morphologic abnormalities, a bone marrow biopsy should be done

- Discontinue TPO-RA in case of reticulin fibrosis grade 3 or collagen fibrosis

- Continue TPO-RA in case of reticulin fibrosis grade 2 and rebiopsy 6 months later [37]

Thrombosis

- ITP is a procoagulant condition (more arterial thrombosis (AT) and venous thromboembolism (VTE))

- TPO-RAs increase the risk of VTE and AT compared to ITP

- Thrombosis not correlated with type, duration, dose of TPO-RA or platelet count

- Splenectomy increases the risk of VTE.

- TPO-RAs do not increase the thrombotic risk in splenectomized patients

- Careful consideration benefits vs. risk of thromboembolism

Rebound thrombocytopenia: more severe thrombocytopenia after drug discontinuation (10\%) due to endogenous TPO deprivation (advised to taper TPORAs and not to withdraw them abruptly)

Fluctuating platelet counts: more frequent with romiplostim

Neutralizing antibody formation to romiplostim (not existing for small molecules): incidence of \pm 6 / 1000, no cross-reaction with endogenous TPO

- If loss of response: test for the formation of antiromiplostim antibodies

Hepatotoxicity: $10 \%$ of patients on eltrombopag develop elevated liver enzymes which occurs more frequently in the first year

- Control of liver function before and each 2 weeks during titration and monthly following achievement of a stable dose

\section{Cataract}

- Recommended to do an ophthalmologic examination in patients with one or more risk factors (older age, steroid use, smoking) and treated with eltrombopag or romiplostim

Induction of malignancy: no indication that the TPO-RAs induce hematological and nonhematological malignancies in ITP

\section{Table 9: Thrombocytopenia in pregnancy}

ITP:

- diagnosis of exclusion (exclude secondary ITP especially SLE and APS)

- more likely if a history of thrombocytopenia before pregnancy

- may present at any point during pregnancy

Gestational thrombocytopenia:

- platelets mostly $>70 \times 10^{9} / \mathrm{L}$

- develops in the late second and third trimester

- not associated with an increase in maternal or fetal complications

Preeclampsia:

- arterial hypertension (systolic >140 and/or diastolic blood pressure $>90 \mathrm{~mm} \mathrm{Hg}$ ), proteinuria (>300 mg/24 h) and thrombocytopenia in 50\% of patients

- thrombocytopenia may preceed other signs

- develops after 20 weeks of gestation

- delivery of the fetus after stabilization of the mother

- platelet transfusions in the setting of severe thrombocytopenia and bleeding

HELLP syndrome:

- Nausea, malaise, upper right quadrant pain or epigastric pain, microangiopathic hemolytic anemia, abnormal liver function (transaminases $>70 \mathrm{U} / \mathrm{L}$ ) and thrombocytopenia

- most frequent in third trimester

- delivery of the fetus after stabilization of the mother

- platelet transfusions in the setting of severe thrombocytopenia and bleeding

Thrombotic trombocytopenic purpura (TTP) and hemolytic uremic syndrome (HUS):

- non-immune microangiopathic hemolytic anemia, thrombocytopenia, and organ impairment (neurological changes more pronounced in TTP and renal changes in HUS)

- TTP most frequent in the second trimester

- $90 \%$ of HUS developing in the postpartum period

- Sometimes difficult to distinguish from hypertensive disorders and HELLP syndrome

- plasma exchange and replacement with fresh frozen plasma

Acute fatty liver of pregnancy: 
- malaise, anorexia, nausea, vomiting, epigastric or right upper quadrant pain, mental status changes and cholestatic liver abnormalities with DIC

- typically occurs in primipara in the third trimester

- delivery of the fetus after stabilization of the mother.

\section{Disclosure statement}

No potential conflict of interest was reported by the authors.

\section{References}

[1] Rodeghiero F, Stasi R, Gernsheimer T, et al. Standardization of terminology, definitions and outcome criteria in immune thrombocytopenic purpura of adults and children: report from an international working group. Blood. 2009;113(11):2386-2393. .

[2] Provan D, Stasi R, Newland AC, et al. International consensus report on the investigation and management of primary immune thrombocytopenia. Blood. 2010;115(2):168-186. .

[3] Cines DB, Bussel JB, Liebman HA, et al. The ITP syndrome: pathogenic and clinical diversity. Blood. 2009;113:6511-6521.

[4] Moulis G, Germain J, Comont T, et al. Newly diagnosed immune thrombocytopenia adults: clinical epidemiology, exposure to treatments, and evolution. Results of the CARMEN multicenter prospective cohort. Am J Hematol. 2017;92(6):493-500.

[5] Arnold DM, Nazy I, Clare R, et al. Misdiagnosis of primary immune thrombocytopenia and frequency of bleeding: lessons from the mcMaster ITP registry. Blood Adv. 2017;1:2414-2420.

[6] Miltiadous O, Hou M, Bussel JB. Identifying and treating refractory ITP: difficulty in diagnosis and role of combination treatment. Blood. 2020;135:472-490.

[7] Despotovic JM, Grimes AB. Pediatric ITP: is it different from adult ITP? Hematology Am Soc Hematol Educ Program. 2018;2018:405-411.

[8] Schifferli A, Holbro A, Chitlur M, et al. A comparative prospective observational study of children and adults with immune thrombocytopenia: 2-year follow-up. Am J Hematol. 2018;93:751-759.

[9] Schoonen WM, Kucera G, Coalson J, et al. Epidemiology of immune thrombocytopenic purpura in the general practice research database. $\mathrm{Br}$ J Haematol. 2009;145(2):235-244. .

[10] Yong M, Schoonen WM, Li L, et al. Epidemiology of paediatric immune thrombocytopenia in the general practice research database. $\mathrm{Br}$ J Haematol. 2010;149 (6):855-864. .

[11] Christiansen CF, Bahmanyar S, Ghanima W, et al. Chronic immune thrombocytopenia in denmark, sweden and norway: the nordic country patient registry for romiplostim. EClinicalMedicine. 2019;14:80-87.

[12] Weycker D, Hanau A, Hatfield M, et al. Primary immune thrombocytopenia in US clinical practice: incidence and healthcare burden in first 12 months following diagnosis. J Med Econ. 2020;23(2):184-192.

[13] Zufferey A, Kapur R, Semple JW. Pathogenesis and therapeutic mechanisms in immune thrombocytopenia (ITP). J Clin Med. 2017;6(2):16-36.
[14] Audia S, Mahévas $M$, Samson $M$, et al. Pathogenesis of immune thrombocytopenia. Autoimmun Rev. 2017;16 (6):620-632. .

[15] Wen R, Wang Y, Hong Y, et al. Cellular immune dysregulation in the pathogenesis of immune thrombocytopenia. Blood Coagul Fibrinolysis. 2020;31(2):113-120.

[16] Li J, Sullivan JA, Ni H. Pathophysiology of immune thrombocytopenia. Curr Opin Hematol. 2018;25 (5):373-381.

[17] Cheloff A, Kuter D, Al-Samkari H. Serum complement levels in immune thrombocytopenia: characterization and relation to clinical features. Res Pract Thromb Haemost. 2020;4(5):807-812.

[18] Provan D. Successful management of primary immune thrombocytopenia. Publisher Evolving medicine Ltd; 2011.

[19] McMillan R, Bussel JB, George JN, et al. Self-reported health-related quality of life in adults with chronic immune thrombocytopenic purpura. Am J Hematol. 2008;83(2):150-154. .

[20] Kruse C, Kruse A, Watson S, et al. Patients with immune thrombocytopenia (itp) frequently experience severe fatigue but is it under-recognized by physicians: results from the itp world impact survey (i-wish). Blood. 2018;132(suppl Supplement 1):2273. .

[21] Cooper N, Kruse A, Kruse C, et al. Results from the itp world impact survey (i-wish): patients with immune thrombocytopenia (itp) experience impaired quality of life (qol) regarding daily activities, social interactions, emotional well-being and working lives. Blood. 2018;132(suppl Supplement 1):4804. .

[22] Janssens A, Lambert C, Bries G, et al.; Primary immune thrombocytopenia in adults. Guidelines for diagnosis and treatment anno 2013 proposed by the belgian hematological society. Belg J Hematol. 2013;4:11-20.

[23] Provan D, Arnold DM, Bussel JB, et al. Updated international consensus report on the investigation and management of primary immune thrombocytopenia. Blood Adv. 2019;3:3780-3817.

[24] Matzdorff A, Meyer O, Ostermann $H$, et al. Immunthrombozytopenie - aktuelle diagnostik und therapie: empfehlungen einer gemeinsamen Arbeitsgruppe der DGHO, ÖGHO, SGH, GPOH und DGTI. Oncol Res Treat. 2018;41(suppl 2):5-36. .

[25] Stasi R. Immune thrombocytopenic purpura: the treatment paradigm. Eur J Haematol. 2009;82:13-19.

[26] Portielje JEA, Westendorp RGJ, Kluin-Nelemans HC, et al. Morbidity and mortality in adults with idiopathic thrombocytopenic purpura. Blood. 2001;97 (9):2549-2554.

[27] Neunert C, Terrell DR, Arnold DM, et al. American society of hematology 2019 guidelines for immune thrombocytopenia. Blood Advances. 2019;3 (23):3829-3866. .

[28] Cheng Y, Wong RSM, Soo YOY, et al. Initial treatment of immune thrombocytopenic purpura with high-dose dexamethasone.. N Engl J Med. 2003;349(9):831-836. .

[29] Sailer T, Lechner K, Panzer S, et al. The course of severe autoimmune thrombocytopenia in patients not undergoing splenectomy.. Haematologica. 2006;91 (8):1041-1045.

[30] Cines DB, Bussel JB. How I treat idiopathic thrombocytopenic purpura (ITP). Blood. 2005;106(7):2244-2251.

[31] Mithoowani S, Gregory-Miller K, Goy J, et al. High-dose dexamethasone compared with prednisone for previously untreated primary immune thrombocytopenia: 
a systematic review and meta-analysis. Lancet Haematol. 2016;3(10):e489-e496. .

[32] Park YH, Yi HG, Kim CS, et al. Clinical outcome and predictive factors in the response to splenectomy in elderly patients with primary immune thrombocytopenia: a multicenter retrospective study. Acta Haematol. 2016;135(3):162-171. .

[33] Janssens A, Dierickx D. Is there a place for rituximab in the management of adult chronic primary immune thrombocytopenia? Belg J Hematol. 2011;2:107-115.

[34] Deshayes S, Khellaf M, Zarour A, et al. Long-term safety and efficacy of rituximab in 248 adults with immune thrombocytopenia: results at 5 years from the french prospective registry ITP-ritux. Am J Haematol. 2019;94 (12):1314-1324. .

[35] Patel V, Mahévas M, Lee S, et al. Outcomes 5 years after response to rituximab therapy in children and adults with immune thrombocytopenia. Blood. 2012;119 (25):5989-5995. .

[36] Janssens A. Romiplostim for the treatment of chronic primary immune thrombocytopenia. Expert Rev Hematol. 2012;5:133-144.

[37] Ghamina W, Cooper N, Rodeghiero F, et al. Thrombopoietin receptor agonists: 10 year later. Haematologica. 2018;104:1112-1123.

[38] Gonzalez-Porras J, Bastida J. Eltrombopag in immune thrombocytopenia: efficacy review and update on drug safety. Ther Adv Drug Saf. 2018;9:263-285.

[39] Jurczak W, Chojnowski C, Mayer J, et al. Phase 3 randomised study of avatrombopag, a novel thrombopoietin receptor agonist for the treatment of chronic immune thrombocytopenia. $\mathrm{Br}$ J Haematol. 2018;183:479-490.

[40] Newland A, Godeau B, Priego V, et al. Remission and platelet responses with romiplostim in primary immune thrombocytopenia: final results from a phase 2 study. $\mathrm{Br} J$ Haematol. 2016;172:262-273.

[41] González-López TJ, Fernández-Fuertes F, Hernández-Rivas JA, et al. Efficacy and safety of eltrombopag in persistent and newly diagnosed ITP in clinical practice. Int $J$ Hematol. 2017;106:508-516.

[42] Kuter D, Newland A, Chong B, et al. Romiplostim in adult patients with newly diagnosed or persistent immune thrombocytopenia (ITP) for up to 1 year and in those with chronic ITP for more than 1 year: a subgroup analysis of integrated data from completed romiplostim studies. $\mathrm{Br} J$ Haematol. 2019;185:503-513.
[43] Neunert CE, Cooper N. Evidence-based management of immune thrombocytopenia: ASH guideline update. Hematology Am Soc Hematol Educ Program. 2018;2018:568-575.

[44] Janssens A, Beguin Y, Lambert C, et al. Treatment-free remission after TPO receptor agonists in adult ITP patients in Belgium. HemaSphere. 2019;3(S1):301. PF 694.

[45] Al-Samkari H, Kuter DJ. Immune thrombocytopenia in adults: modern approaches to diagnosis and treatment. Semin Thromb Hemost. 2020;46:275-288.

[46] Bussel J, Arnold D, Boxer M, et al. Long-term fostamatinib treatment of adults with immune thrombocytopenia during the phase 3 clinical trial program. Am J Haematol. 2019;94:546-553.

[47] Cooper N, Ghamina W. Immune thrombocytopenia. N Engl J Med. 2019;381:945-955.

[48] Robak T, Kaźmierczak M, Jarque I, et al. Phase 2 multiple-dose study of an FcRn inhibitor, rozanolixizumab, in patients with primary immune thrombocytopenia. Blood Adv. 2020;4:4136-4146.

[49] Newland A, Sanchez-Gonzalez B, Rejto L, et al. Phase 2 of efgartigimod, a novel FcRn antagonist, in adult patients with primary immune thrombocytopenia. Am J Hematol. 2020;95:178-187.

[50] Witkowski M, Witkowski M, Robak T. Autoimmune thrombocytopenia: current treatment options in adults with a focus on novel drugs. Eur J Haematol. 2019;103:531-541.

[51] Stavrou E, Mc Crae K. Immune thrombocytopenia in pregnancy. Hematol Oncol Clin North Am. 2009;23:1299-1316.

[52] Khellaf M, Lousteau V, Bierling P, et al. Trombopenie et grossesse. Rev Med Interne. 2012;33:446-452.

[53] Cines DB, Levine LD. Thrombocytopenia in pregnancy. Blood. 2017;130:2271-2277.

[54] Care A, Pavord S, Knight M, et al. Severe primary autoimmune thrombocytopenia in pregnancy: a national cohort study. BJOG. 2018;125:604-612.

[55] Pavord S, Thachil J, Hunt BJ, et al. Practical guidance for the management of adults with immune thrombocytopenia during the COVID-19 pandemic. Br J Haematol. 2020;189:1038-1043.

[56] Ekstrand C, Linder $M$, Cherif $H$, et al. Patient characteristics when starting treatment and patterns of treatment in adults with chronic immune thrombocytopenia. Blood. 2019;30:350-356.

[57] Loos J, Beckers $M$, Beckers $V$, et al. Vaccinations in patients with hematological malignancies and asplenia without stem cell transplantation: recommendations from the advisory board on vaccination of the belgian hematological society. Belg J Hematol. 2020 accepted for publication 